\title{
A two step model for senescence triggered by a single critically short telomere
}

\author{
Pauline Abdallah ${ }^{1,}{ }^{,}$, Pierre Luciano ${ }^{2,{ }^{*}}$, Kurt W. Runge ${ }^{3}$, Michael Lisby ${ }^{4}$, Vincent Géli $^{2}$, Eric \\ Gilson $^{1, \# \text {, and M. Teresa Teixeira }}{ }^{1}$ \\ ${ }^{1}$ LBMC, UMR 5239, CNRS/ ENS Lyon, Université Lyon 1, Ecole Normale Supérieure, 46 allée \\ d'Italie, F-69364 Lyon Cedex 07, France \\ ${ }^{2}$ CNRS-IBSM-IGC, 31 chemin Joseph Aiguier, F-13402 Marseille cedex 20, France \\ ${ }^{3}$ Cleveland Clinic Foundation, Lerner Research Institute, 9500 Euclid Avenue, NE20, Cleveland, \\ $\mathrm{OH} 44195$ \\ ${ }^{4}$ Department of Biology, University of Copenhagen, Ole Maaløes Vej 5, DK-2200 Copenhagen N, \\ Denmark
}

\begin{abstract}
The telomeres protect chromosome ends from fusion and degradation ${ }^{1}$. In the absence of a specific telomere elongation mechanism, their DNA progressively shortens with every round of replication leading to replicative senescence ${ }^{2}$. Here, we show that telomerase-deficient cells bearing a single very short telomere senesce earlier, demonstrating that length of the shortest telomere is a major determinant of the onset of senescence. We further show that Mec1p/ATR recognises specifically the single very short telomere causing the accelerated senescence. Strikingly, before entry into senescence, cells divide for some generations despite a complete erosion of their shortened telomeres. This pre-senescence growth requires RAD52 and MMS1 and there is no evidence for major inter-telomeric recombination. We propose that, in the absence of telomerase, a very short telomere is first maintained in a pre-signalling state by a RAD52/MMS1 dependent pathway and then switches to a signalling state leading to senescence through a Mec1p-dependent checkpoint.
\end{abstract}

In mouse, the presence of a minimal set of very short telomeres is sufficient to trigger replicative senescence ${ }^{3}$. Nevertheless, it is still unclear whether one single very short telomere is enough and if so, what is the critical telomere length that signals senescence. To address this question we created a telomerase-negative budding yeast strain where a single telomere was engineered to be suddenly extremely reduced without affecting the integrity of its end (Figure 1). Specifically, we constructed a diploid strain lacking one copy of the telomerase RNA gene $T L C 1$ and containing a truncated pair of VII-L telomeres devoid of any natural subtelomeric sequences to focus on the role of the minimal functional unit of telomere - the telomeric repeats. The modified VII-L telomere, used previously to monitor various aspects of telomere function ${ }^{4,5}$, contains internal telomeric repeats flanked by recognition sites for a recombinase. After sporulation, inducing the recombinase during

\footnotetext{
${ }^{\#}$ Correspondence should be addressed to EG : eric.gilson@ens-lyon.fr.

* These authors contributed equally to this work
} 
germination results in excision without affecting the integrity of the chromosome end. Telomerase-negative colonies are selected by means of the marker used to delete $T L C 1$, which is only expressed in haploid cells ${ }^{6}$. Three sets of strains were created containing an increasing number of internal telomeric repeats: a shortening of about 100-120 bp is achieved with strains named 1 or 2 blocks, compared to the control 0 block strains (Figure 1c).

When several independent control spores ( 0 block) are serially spotted to follow senescence, we observe that, as expected, cell growth is severely impaired at the $3^{\text {rd }}$ passage (Figure 2a). However, cells containing a single short telomere ( 1 or 2 blocks), loose the capacity to form colonies at the $2^{\text {nd }}$ passage. Glucose grown colonies that do not shorten telomeres did not show any difference in the onset of senescence (not shown). Median viability curves based on cell number and colony size revealed that the shorter the VII-L telomere, the sooner cells lost viability (Figure 2b, Methods and Supplemental Table 1). Morphologic inspection and DNA staining shows that a large fraction of cells containing a very short telomere are arrested in the G2/M cell cycle phase (Figure 2c), indicating that the growth arrest triggered by the very short telomere is similar to bona fide senescence. Thus, the number of generations until a telomerase-negative cell senesces is reduced by shortening a single telomere.

To monitor the timing of the onset of senescence more precisely, we grew cells continuously in liquid culture and measured the number of population doublings undergone by each strain every 24h (Figure 2d). As with bona fide senescence, culture growth rate decreases then increases with the appearance of rapid growing survivors ${ }^{7}$. We found that shortening a single telomere accelerates the onset of senescence by approximately 15 population doublings (from 60 to 45 generations, Figure 2e). The 45 generations of growth for the strains bearing a short telomere was surprising, given that these strains initially have $\sim 100$ bp of $\mathrm{TG}_{1-3}$ repeats on the VIIL telomeres (Figure 1) that should shorten by 3 to $4 \mathrm{bp} /$ generation ${ }^{4}$, suggesting that all repeats would be gone in 30 generations. Most of the spores that lost 2 blocks of repeats grow for 45 generations, indicating that the truncated VII-L telomere is somehow maintained in functional state in pre-senescent cells and/or that a second event is required to trigger senescence.

To test whether a second event contributes to senescence, we followed growth of a given spore in 8 independent senescence assay replicates and found that the viability profiles were almost identical between replicates from control spores (Supplementary Figure 1a), similar to previous work ${ }^{8}$. Thus, the timing of the second event is apparently fixed, raising the possibility that replicative senescence is triggered by a structural switch of a single very short telomere from a pre-signalling state to a signalling state of senescence.

To determine whether the structure of the very short telomere switches to a senescencesignaling state, telomere length of telomere VIIL was measured by telomere-PCR ${ }^{9}$. In addition to the modified VIIL telomere, the majority of telomeres in this strain bear a subtelomeric repeat called $\mathrm{Y}^{\prime}$ whose length can also be measured ${ }^{10}$. We observed that the $\mathrm{Y}^{\prime}$ and modified-VIIL telomeres of 0 block strains progressively shortened (Figure 3a). As expected, the length of the modified-VIIL telomere was shorter than the other telomeres in 
the spores with 1 and 2 blocks. Strikingly, the length of the very short telomere with 1 or 2 blocks was approximately constant in the original colony, the replicating cells in passage 1 and the largely senescent population in passage 2 and 3. Thus, the switch to a senescencesignaling state does not seem to involve a further reduction in the size of the very short telomere. To determine the structures of the senescence- pre-signaling and signaling telomeres, modified-VIIL telomeres were cloned and sequenced (Figure 3b). Surprisingly, many DNA ends did not contain any telomeric repeats even though these telomeres were cloned from colonies just after germination, when the majority of cells are viable (Figure 3a passage 1).

To investigate how could cells sustain cell proliferation despite the complete or nearly complete loss of telomere sequence, we checked whether the pre-senescent cells had adapted to a non-reparable double strand break ${ }^{11}$. When Ptc2p, a critical phosphatase involved in this process ${ }^{12}$ was deleted, we saw no reduction in cell viability (Supplementary Figure 1b-c and Supplementary Table 1). Thus, adaptation is unlikely to have a key role in the viability of cells bearing the very short telomere.

In order to test whether the senescence- pre-signalling telomere is maintained by homologous recombination, we examined the role of $R A D 52$. In both the 0 and 2 block conditions, viability of rad52 $\Delta$ spores was severely compromised compared to wild type colonies (Figure 3c, Supplementary Figure 1d and Supplementary Table 1), in agreement with previous work ${ }^{7}$. Moreover, the loss of viability correlated with the increased accumulation of G2/M cells (Supplemental Figure 1e). Thus, the absence of RAD52 and telomerase activity severely reduced the number of divisions that cells can undergo before to enter into senescence. Both RAD52 and rad52 $\Delta$ cells had similar telomere lengths (Figure 3a right panels), despite the fact that $\mathrm{rad} 52 \Delta$ cells have undergone fewer population doublings. Thus, the role of Rad52p in pre-senescence does not seem to involve a major modification of the length of the very short telomere.

To determine whether RAD52 supports cell growth by promoting recombination between the very short telomere and the other telomeres of the nucleus, the sequences of the modifiedVIIL telomeres from $R A D 52 t l c 1 \Delta$ strains where examined for the addition of novel sequences. Only two of the 35 very short cloned telomeres from pre-senescent cells acquired divergent sequences (Figure 3b), similar to the frequency observed in previous work for a median length telomere ${ }^{13}$. Thus, the maintenance of the very short telomere in a presenescent state can not be simply explained by an increased rate of inter-telomeric or unequal intra-telomeric exchanges. These data also show that the recombination-based mode of telomere maintenance occurring in post-senescence survivors ${ }^{7,14}$ is not yet operational in pre-senescent cells.

Rad52p functions in several pathways of homologous recombination including the repair of double-strand breaks and the repair of stalled replication forks ${ }^{15}$. To determine if one of these pathways is involved in the maintenance of the very short telomere, we then tested the effect of mutating $M M S 1$, a gene involved in the repair of stalled forks but not of double strand breaks ${ }^{16-19}$. We see that $m m s 1 \Delta$ had an effect similar to the one of $\operatorname{rad} 52 \Delta$ (Figure 3d, Supplemental Figure 1f and Supplemental Table 1). Therefore, a function common to 
MMS1 and RAD52 seems to be required to maintain viability of pre-senescent cells. Because both genes are required to repair stalled replication forks, we speculate that very short telomeres are particularly difficult to replicate and require homologous recombination to complete their replication.

Next, we asked whether the (PI)-3-kinase-related protein kinase Mec1p/ATR is required for accelerated senescence in the presence of a very short telomere. In order to compensate for the lack of the $M E C l$ essential function, SMLI was also deleted in the starting diploids strains ${ }^{20}$. Compared to $M E C 1 S M L 1$ strains, we observed that loss of viability of mecl $\Delta$ $\operatorname{smll} \Delta$ strains was delayed approximately one passage in the absence of a G2/M cell cycle arrest (Figure 4a, Supplementary Figure 1g-h and Supplementary Table 1) confirming previous observations involving Mec1p in the signaling of senescence ${ }^{8,21}$.

No statistically significant differences in times of senescence were observed between the $M E C l S M L 1$ and $m e c 1 \Delta \operatorname{smll} \Delta$ with very short telomeres (2 block strains, Figure 3a and Supplemental Figure 1g) in the first two passages. However, most mecl $\Delta \operatorname{smll} \Delta$ clones with a very short telomere are still viable after the $3^{\text {rd }}$ passage and their viability curves resemble the mec $1 \Delta \operatorname{smll} \Delta$ control strains with a 0 block telomere. This ability of cells to form colonies was not due to post-senescence survival since after the $4^{\text {th }}$ passage most cells stop growing in the absence of a marked G2/M arrest (Supplemental Figure 1h). Therefore, lack of Mec1p and Sml1p bypasses the accelerated senescence due to the presence of a very short telomere. We conclude that a senescence-signaling telomere acquires the property to activate Mec1p.

In order to test whether Mec1p senses the senescence-signalling telomere through a direct binding, we generated telomerase-negative spores with tagged versions of Mec1p (Mec1HA) and carrying a single shortened telomere. Chromatin immunoprecipitation showed that Mec1p was enriched at another chromosomal telomere in pre-senescent cells, but the enrichment at the very short telomere is about 2 times more efficient (Figure 4b). This supports the idea that Mec1p interacts physically with the signalling telomere and by this way senses and transduces a specific signal emanating from it into a G2/M cell cycle arrest.

Tel1 p binds to short telomeres ${ }^{5,22-24}$ but its impact on senescence is still elusive ${ }^{8,21,25}$. To get insights into Tel1p's role in senescence, we introduced the tell $\Delta$ mutation in our system. We noticed that TEL1 0 block cells descending from a TEL1/tell $\triangle$ heterozygote were systematically senescing earlier than the ones descending from TEL1/TEL1 diploids, allowing only two passages to be analysed. Similar to previously described ${ }^{21,25}$, tell $\Delta$ spores senesced later than $w t$ (TEL1) sister spores even in the presence of a very short telomere (Figure 4c, Supplementary Figure 1i and Supplementary Table 1), in contrast to the $m e c 1 \Delta$ smll $\Delta$ cells (Figure $4 a$ ). Thus, TEL1 promotes replicative senescence but its loss did not abolish the accelerated senescence caused by a very short telomere.

We also generated telomerase-negative spores with tagged versions of Tel1p (Tel1-HA) and carrying the single shortened telomere. Chromatin immunoprecipitation shows that, in agreement with previous results ${ }^{5,23,24}$, Tel1p efficiently binds a very short telomere, in the presence or absence of Mec1p (Figure 4d). The enrichment for Tel1p at the short telomere 
was much more pronounced compared to Mec1p. This may result from a difference in immunoprecipitability between the two proteins. Alternatively this could reflect the possibility that Tel1p binds in pre-senescent dividing cells, whereas Mec1p binds only in senescent non-dividing cells.

The Tel1-HA strong association with the shortened telomere before the first passage also indicates that Tel1p association does not result in senescence. These data, with recent work showing that a single double-strand break does not induce a Tel1p-mediated checkpoint ${ }^{26}$, make Tel1p an unlikely candidate for the checkpoint protein that induces senescence.

Our results show that a single very short telomere determines the onset of replicative senescence. Moreover, the dissection of the events occurring at this single short telomere before and during senescence reveals a two-step mechanism (Figure 5). First, the very short telomere is maintained in a senescence- pre-signalling state through a recombination pathway involving Rad52p and Mms1p that preserves the sequence of individual telomeres. Then, it switches to a signalling state of senescence where it induces cell cycle arrest by binding to Mec1p.

Intriguingly, we have been unable to detect any significant telomere length differences that could account for the switch from the pre-signalling to the signalling state of senescence. Moreover, some senescence- pre-signalling telomeres might have completely lost their telomeric repeats, suggesting that capping functions can be epigenetically maintained for few generations after a complete telomeric DNA erosion ${ }^{27}$. In any case, we show that this pre-senescent state is maintained thanks to an activity driven by RAD52 and MMS1 that does not result in inter-telomeric recombination or intra-telomeric unequal recombination. In further agreement with a specific role of Rad52p in telomere maintenance, the accompanying paper ${ }^{28}$ shows that Rad52p is rapidly recruited to the very short telomere in telomerase negative cells. Overall, we propose that a very short or even an eroded telomere can be maintained for some generations in a pre-signalling state of senescence through sister chromatid recombination. Since this pathway is usually found in the repair of arrested replication forks, we suggest that a very short telomere could be sensitive to fork progression. Both the specificity of the phenotype of $M E C l$ deletion in the cells containing a very short telomere and the recognition of the very short telomere by Mec1p-Ddc2p and Replication Protein A (this work and the accompanying paper ${ }^{28}$ suggest that the primary signal triggering senescence could be a long stretch of single-stranded DNA.

The relative contribution of ATR/Mec1p and ATM/Tel1 to telomere dysfunction induced checkpoints remains yet to be defined in mammalian models. The fact that checkpoint activation in intestinal stem cells harboring dysfunctional telomeres involves Exo1-mediated activation of ATR/Mec1 $1 \mathrm{p}^{29}$ and that fibroblasts in the dermis of baboons rarely contain more than one telomere damage focus, even in very old animals ${ }^{30}$, suggest that, like we show here in yeast, mammalian cells have the ability to signal a single critically short telomere through an ATR/Mec1p checkpoint. We propose that this single telomere checkpoint reflects a conserved propriety of eukaryotic cells that contributes to the control of genome stability and cellular homeostasis. 


\section{Methods}

\section{Strains}

All strains constructed for this work are in the W303 background and are listed in Supplementary information. All strains were generated from lev187, lev189 and lev220 ${ }^{4}$, a wild type W303 strain bearing the tlc1 $1::$ PaNatMX4, constructed as in reference ${ }^{6}$ and strains from the T. Petes ${ }^{31}$ and M-P. Longhese ${ }^{32}$ laboratories. Details are available upon request.

\section{Obtaining a short telomere in a telomerase-negative context}

Diploids are sporulated in $2 \%$ potassium acetate plates for 3 days at $30^{\circ} \mathrm{C}$ until sporulation efficiency reaches more than $20 \%$. The mixture is then resuspended in 100 microliters of water and equal amounts of cells are used to inoculate $2 \mathrm{ml}$ of YPGal/Raff (2\% Galactose, $1 \%$ Raffinose) or YPD liquid media at about $1-5.10^{6}$ tetrads $/ \mathrm{ml}$. After $6 \mathrm{~h}$ of germination at $30^{\circ} \mathrm{C}$, several dilutions of the germination mixture are plated in YPD supplemented with nourseothricin. Colonies are allowed to form for $40 \mathrm{~h}$ at $30^{\circ} \mathrm{C}$. Only 1 to $10 \%$ of cells germinated in Galactose-containing media form colonies, whereas the colony formation capacity is $80-100 \%$ when cells are germinated in YPD. Since the strains containing no additional telomeric repeats ( 0 block) show the same 1 to $10 \%$ germination, the process of telomere excision does not appear to alter germination. Colonies on plates of similar colony density (typically 500-1000 colonies per plate) are then tested for uracil prototrophy or any other marker by streaking a very small portion of the colony on appropriate media. Identification of spores containing the HA tags, the $\operatorname{smll} \Delta$ and $t e l l \Delta$ alleles was performed by PCR (oligonucleotide sequences available upon request).

\section{Semi-quantitative senescence assay}

Nourseothricine resistant, ura $^{-}$colonies are resuspended in water in microtiter plates, optical density at $600 \mathrm{~nm}$ is measured, cell concentrations are determined based on a standard curve and dilutions are performed to obtain suspensions of equal cell concentration. In most experiments, this initial concentration is $400000 \mathrm{cells} / \mathrm{ml}$. When the spore colonies were very small (e.g. rad52 $\Delta$ cells), a lower initial concentration was used. In all cases, the concentration of the wild type controls and mutant strains within an experiment were equal, and cells were plated at the same time. Spots of 10x serial dilutions of these suspensions are grown two days at $30^{\circ} \mathrm{C}$ in YPD supplemented with Nourseothricin. The most concentrated spots (representing the mixture of many colonies) are then resuspended, diluted to a known concentration and re-spotted on plates as previous for the next serial passage.

Telomerase-negative cells with short telomeres exhibit two phenotypes: reduced plating efficiency and slow colony growth. To quantify both phenotypes simultaneously, we calculated a viability index for each spore colony that is based on both the number of colonies in the spot with the highest dilution and the area covered by each isolated colony (which is reduced for slow growing cells). Thus, the viability index is the area (in $\mathrm{mm}^{2}$ ) of the colonies from the spot containing isolated colonies (measured using Image ${ }^{33}$ ) multiplied by the dilution factor of the spot normalised by the initial concentration of cells. Passage 1 viability index corresponds to the growth potential of cells contained in the spore- 
colony; passage 2 viability index corresponds to cells of colonies from passage 1 , etc... Within the same experiment and passage, differences in the set of viabilities indexes between different genotypes are statistically tested with the Wilcoxon rank sum test (MannWhitney) using Package R v2.6.1 $1^{34}$ or XLstat (v2008.1.01).

\section{Microscope analysis}

Exponentially growing cells were fixed with formaldehyde 3,7\% for 45 minutes, treated with Zymolyase at $2,5 \mathrm{mg} / \mathrm{ml}$ for 20 minutes at $37^{\circ} \mathrm{C}$ and applied to slides coated with Polylysine $0,1 \%$. The slides were washed with PBS $1 \mathrm{X}$ and then incubated in $0,5 \mathrm{mg} / \mathrm{ml}$ of DAPI for $1 \mathrm{~h}$. After a brief washing and fixation with Ethanol 100\%, slides were mounted using Dakocytomakon (Dako, Denmark). Images were acquired at 1000 magnification using a Zeiss Axioplan, and images were captured with CCD camera (Photometrix sensys) and processed with Metamorph software. Single unbudded cell with one nucleus and a mother cell with a small bud were designated as a G1/S cell. A mother cell with an equal sized daughter and nucleus at budneck was designated as a G2/M cell. For every time point at least 50 cell-bodies were counted. Because of their poor growth, for $\mathrm{rad} 52 \Delta$ strains, cells from fresh spore formed colonies were directly resuspended in water und observed using a visible light microscope. Comparison between daughter and mother cell sizes was used to estimate the percentage of cells in G2/M.

\section{Chromatin immunoprecipitations}

Chromatin immunoprecipitations of MEC1-HA and TEL1-HA expressing strains were performed as described ${ }^{35}$ using previously described primers ${ }^{5}$.

\section{DNA analysis}

Telomere-PCR and subsequent telomere cloning and sequence analysis was performed as described ${ }^{13}$ using oligonucleotides oT100 ( $5^{\prime}$-CTGTAGGGCTAAAGAACAGGG-3'), oT155 (5'-GGAACTTCGGAATAGGAACTTCAAGACTCGAGAGATCCC-3') and oT157 (5'-ACCAAGTTGATGAGCAAGGG-3'). The oT100 oligonucleotide specifically amplifies long $\mathrm{Y}^{\prime}$ telomeres ${ }^{10}$. Sequence reactions were performed by Fasteris SA (Geneva, Switzerland). To estimate length of the pure $\mathrm{TG}_{1-3}$ tract from the mobility of telomere-PCR we first deducted the subtelomeric region and the oligonucleotide length and we applied a correction. This latter was estimated by comparing the mobility of telomere-PCR products with Southern Blot of the same DNA or by estimating restriction fragment size after cloning or by direct sequencing of resulting plasmids. Corrections applied were for oT155: $y=0,97 x$ -83 , oT157: $y=0,87 x-18,7$ and oT100: $y=0,95 x-98$ where $x$ is the median size of the telomere band in bp. These measurements were valid for telomere-PCR reactions performed at the same time and migrated in the same gel. Sequences of cloned telomeres are available upon request.

\section{Supplementary Material}

Refer to Web version on PubMed Central for supplementary material. 


\section{Acknowledgments}

We would like to thank R. Wellinger and L. Rudolph for fruitful suggestions, G. Yvert and C. Lopes for advice in statistical analysis and A. Jacquier to share unpublished material. We also thank T. Petes, M. P. Longhese and S. Marcand for strains and plasmids. PA gratefully acknowledges the financial support of the Lebanese National council for Scientific Research (CNRSL) and the Association pour la Recherche sur le Cancer (ARC). This work was supported by La Ligue Contre le Cancer (EG and VG, équipes labellisées), the INCa programs TELINCA and TELOFUN (EG and VG), The Danish Agency for Science, Technology and Innovation (ML), the Villum Kann Rasmussen Foundation (ML), and the National Institutes of Health (KR).

\section{References}

1. Palm W, de Lange T. How Shelterin Protects Mammalian Telomeres. Annual review of genetics. 2008; 42:301-334.

2. Gilson E, Geli V. How telomeres are replicated. Nat Rev Mol Cell Biol. 2007; 8:825-838. [PubMed: 17885666]

3. Hemann MT, Strong MA, Hao LY, Greider CW. The shortest telomere, not average telomere length, is critical for cell viability and chromosome stability. Cell. 2001; 107:67-77. [PubMed: 11595186]

4. Marcand S, Brevet V, Gilson E. Progressive cis-inhibition of telomerase upon telomere elongation. Embo J. 1999; 18:3509-3519. [PubMed: 10369690]

5. Sabourin M, Tuzon CT, Zakian VA. Telomerase and Tel1p preferentially associate with short telomeres in S. cerevisiae. Mol Cell. 2007; 27:550-561. [PubMed: 17656141]

6. Decourty L, et al. Linking functionally related genes by sensitive and quantitative characterization of genetic interaction profiles. PNAS. 2008 in press.

7. Lundblad V, Blackburn EH. An alternative pathway for yeast telomere maintenance rescues est1senescence. Cell. 1993; 73:347-360. [PubMed: 8477448]

8. Enomoto S, Glowczewski L, Berman J. MEC3, MEC1, and DDC2 Are Essential Components of a Telomere Checkpoint Pathway Required for Cell Cycle Arrest during Senescence in Saccharomyces cerevisiae. Mol Biol Cell. 2002; 13:2626-2638. [PubMed: 12181334]

9. Forstemann K, Hoss M, Lingner J. Telomerase-dependent repeat divergence at the $3^{\prime}$ ends of yeast telomeres. Nucleic acids research. 2000; 28:2690-2694. [PubMed: 10908324]

10. Louis EJ. The chromosome ends of Saccharomyces cerevisiae. Yeast. 1995; 11:1553-1573. [PubMed: 8720065]

11. Sandell LL, Zakian VA. Loss of a yeast telomere: arrest, recovery, and chromosome loss. Cell. 1993; 75:729-739. [PubMed: 8242745]

12. Leroy C, et al. PP2C phosphatases Ptc 2 and Ptc3 are required for DNA checkpoint inactivation after a double-strand break. Mol Cell. 2003; 11:827-835. [PubMed: 12667463]

13. Teixeira MT, Arneric M, Sperisen P, Lingner J. Telomere Length Homeostasis Is Achieved via a Switch between Telomerase- Extendible and -Nonextendible States. Cell. 2004; 117:323-335. [PubMed: 15109493]

14. Teng SC, Zakian VA. Telomere-telomere recombination is an efficient bypass pathway for telomere maintenance in Saccharomyces cerevisiae. Molecular \& Cellular Biology. 1999; 19:8083-8093. [PubMed: 10567534]

15. Krogh BO, Symington LS. Recombination proteins in yeast. Annual review of genetics. 2004; 38:233-271.

16. Duro E, Vaisica JA, Brown GW, Rouse J. Budding yeast Mms 22 and Mms1 regulate homologous recombination induced by replisome blockage. DNA Repair (Amst). 2008; 7:811-818. [PubMed: 18321796]

17. Hryciw T, Tang M, Fontanie T, Xiao W. MMS1 protects against replication-dependent DNA damage in Saccharomyces cerevisiae. Mol Genet Genomics. 2002; 266:848-857. [PubMed: 11810260]

18. Ui A, et al. Activation of a novel pathway involving Mms1 and Rad59 in sgs1 cells. Biochem Biophys Res Commun. 2007; 356:1031-1037. [PubMed: 17399687] 
19. Zaidi IW, et al. Rtt101 and Mms1 in budding yeast form a CUL4(DDB1)-like ubiquitin ligase that promotes replication through damaged DNA. EMBO Rep. 2008; 9:1034-1040. [PubMed: 18704118]

20. Zhao X, Muller EG, Rothstein R. A suppressor of two essential checkpoint genes identifies a novel protein that negatively affects dNTP pools. Mol Cell. 1998; 2:329-340. [PubMed: 9774971]

21. Ijpma AS, Greider CW. Short Telomeres Induce a DNA Damage Response in Saccharomyces cerevisiae. Mol Biol Cell. 2003; 14:987-1001. [PubMed: 12631718]

22. Bianchi A, Shore D. Increased association of telomerase with short telomeres in yeast. Genes Dev. 2007; 21:1726-1730. [PubMed: 17639079]

23. Arneric M, Lingner J. Tel1 kinase and subtelomere-bound Tbf1 mediate preferential elongation of short telomeres by telomerase in yeast. EMBO Rep. 2007

24. Hector RE, et al. Tel1p preferentially associates with short telomeres to stimulate their elongation. Mol Cell. 2007; 27:851-858. [PubMed: 17803948]

25. Ritchie KB, Mallory JC, Petes TD. Interactions of TLC1 (which encodes the RNA subunit of telomerase), TEL1, and MEC1 in regulating telomere length in the yeast Saccharomyces cerevisiae. Molecular \& Cellular Biology. 1999; 19:6065-6075. [PubMed: 10454554]

26. Mantiero D, Clerici M, Lucchini G, Longhese MP. Dual role for Saccharomyces cerevisiae Tel1 in the checkpoint response to double-strand breaks. EMBO Rep. 2007

27. Sadaie M, Naito T, Ishikawa F. Stable inheritance of telomere chromatin structure and function in the absence of telomeric repeats. Genes Dev. 2003; 17:2271-2282. [PubMed: 12952894]

28. Khadaroo B, et al. The DNA damage response at eroded telomeres and tethering to the nuclear pore complex. Nat Cell Biol. (in press).

29. Schaetzlein S, et al. Exonuclease-1 deletion impairs DNA damage signaling and prolongs lifespan of telomere-dysfunctional mice. Cell. 2007; 130:863-877. [PubMed: 17803909]

30. Jeyapalan JC, Ferreira M, Sedivy JM, Herbig U. Accumulation of senescent cells in mitotic tissue of aging primates. Mech Ageing Dev. 2007; 128:36-44. [PubMed: 17116315]

31. Mallory JC, Petes TD. Protein kinase activity of Tel1p and Mec1p, two Saccharomyces cerevisiae proteins related to the human ATM protein kinase. Proc Natl Acad Sci U S A. 2000; 97:1374913754. [PubMed: 11095737]

32. Longhese MP, Paciotti V, Neecke H, Lucchini G. Checkpoint proteins influence telomeric silencing and length maintenance in budding yeast. Genetics. 2000; 155:1577-1591. [PubMed: 10924458]

33. Abramoff MD, Magelhaes PJ, Ram SJ. Image Processing with ImageJ. Biophotonics International. 2004; 11:36-42.

34. Ihaka R, Gentleman R. R: a language for data analysis and graphics. J Comp Graph Stat. 1996; 5:299-314.

35. Tresaugues L, et al. Structural characterization of Set1 RNA recognition motifs and their role in histone H3 lysine 4 methylation. J Mol Biol. 2006; 359:1170-1181. [PubMed: 16787775]

36. Marcand S, Gilson E, Shore D. A protein-counting mechanism for telomere length regulation in yeast. Science. 1997; 275:986-990. [PubMed: 9020083] 
a

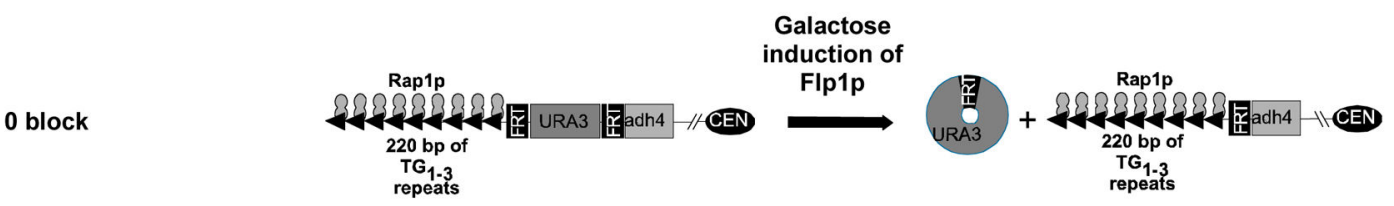

1 block
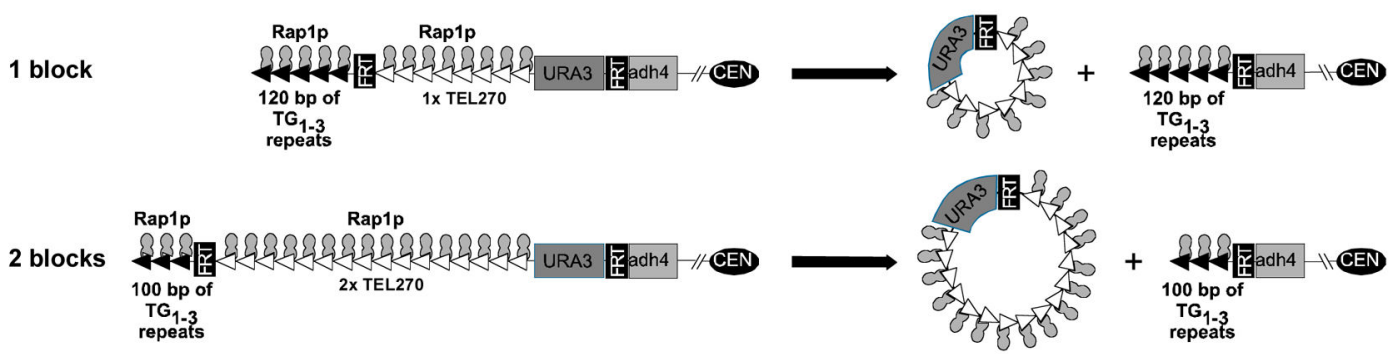

b

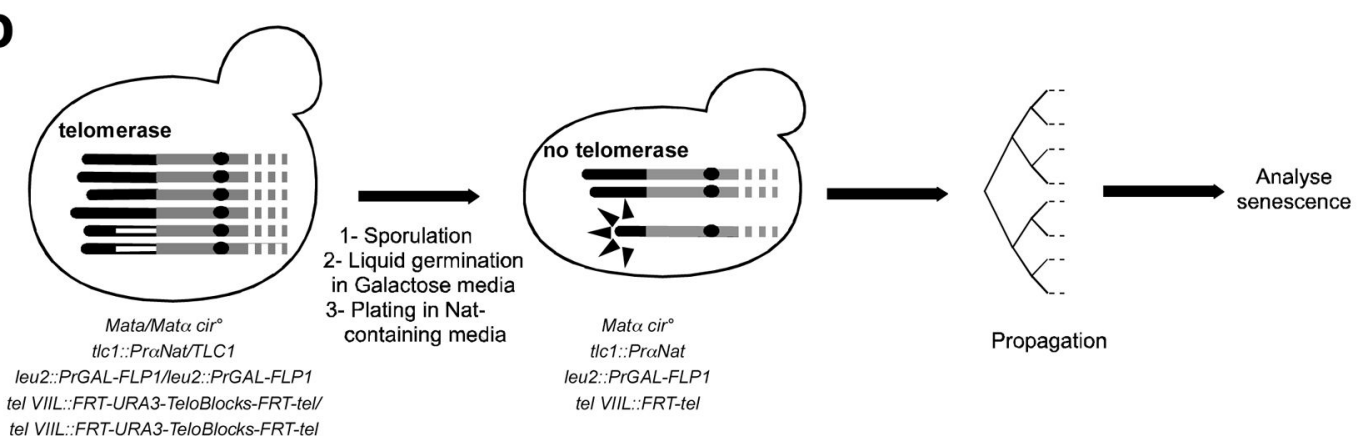

C

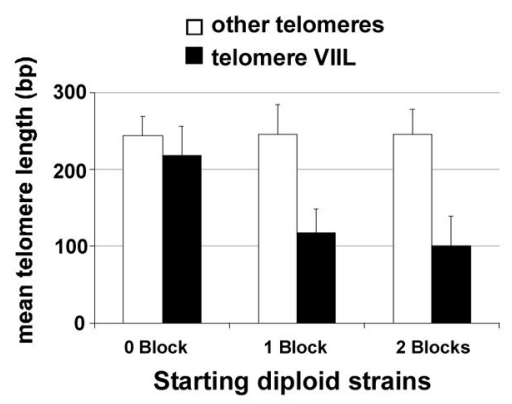

Figure 1. Experimental system

a: We used the artificial telomere constructs first described in reference ${ }^{4}$. The left arm of telomere VIIL is replaced by an artificial telomere at the $A D H 4$ locus. Internal telomeric repeats are introduced in the proximity of the new extremity and counted by telomerase through the Rap1p-counting mechanism ${ }^{36}$. As a consequence, the number of distal telomeric repeats is reduced. The internal telomeric repeats are flanked by an URA3 selective marker and two "Flipase-Recognition Targets" (FRT). Upon expression of the sequence-specific recombinase Flp1p, a circle containing the internal telomeric repeats and $U R A 3$ is excised, leaving a short telomere. Three sets of strains are constructed: " 0 block" that serve as 
control; "1 block" containing 270 bp of internal TG1-3; and "2 blocks", containing two blocks of 270 bp of TG1-3 repeats. b: To obtain the shortening in a telomerase-negative context we created a set of diploid strains homozygous for the modified telomere VII-L $(0,1$ or 2 blocks) and heterozygous for the telomerase RNA (TLC1/tlc1 $\Delta)$. This latter is deleted using a cassette expressing the nourseothricine resistance marker under the control of an alpha-specific promoter ${ }^{6}$. These strains also contain the FLP1 gene under the control of the galactose-inducible promoter (GAL10). Meiotic products of these diploids are germinated in the presence of galactose (or glucose as a control) and plated on nourseothricin-containing medium. The resulting colonies are then checked for their ability to grow on media lacking uracil. Nourseothricin resistant colonies unable to grow in medium lacking uracil are deleted for telomerase, have excised and lost a circle of telomeric $\mathrm{TG}_{1-3}$ repeats and have a telomere tract less than half the size of the average telomere. c: Telomere length of starting diploids (yT136, yT137 and yT138, see Supplementary information) was estimated by telomere-PCR using an oligonucleotide targetting the telomere-proximal/FRT region of telomere VII-L (oT155) and an oligonucleotide targeting a subtelomeric element, the long $\mathrm{Y}^{\prime}$ element ${ }^{10}$, that is conserved in many telomeres of S. cerevisiae (oT100). See Methods for details. Error bars correspond to standard deviation $n=3$ for all samples of other telomeres and $n>4$ for telomere VII-L samples. 
a
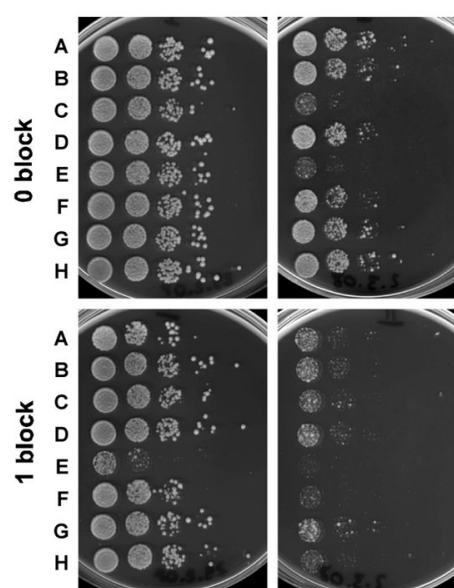

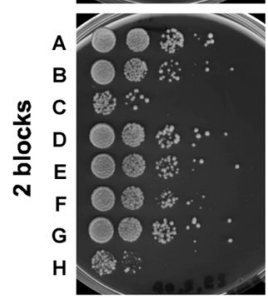

passage 1

d

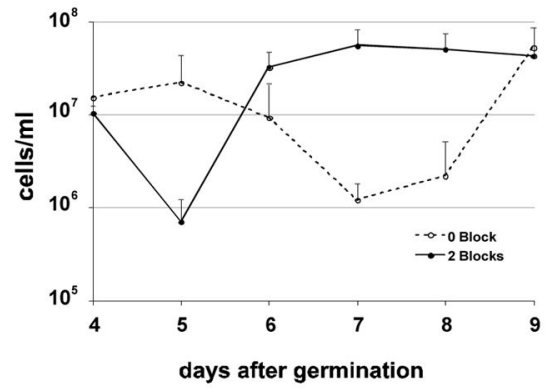

b
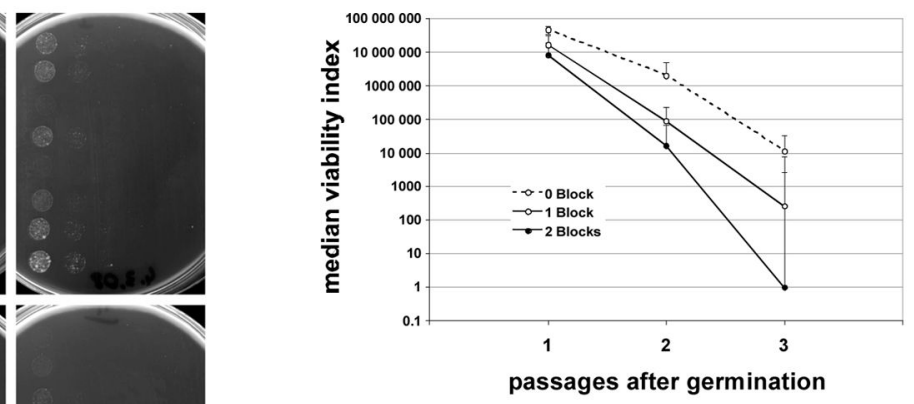

C

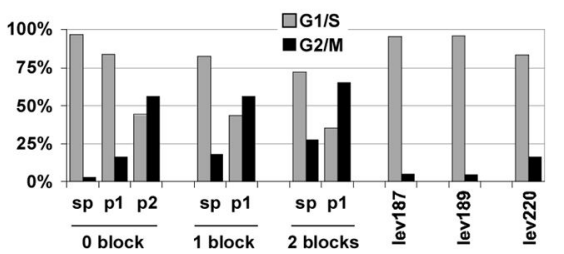

$\mathbf{e}$

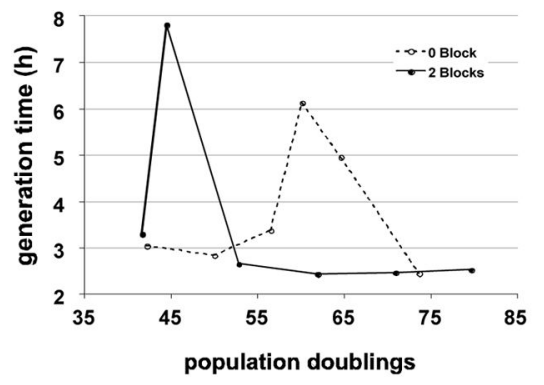

Figure 2. Senescence is advanced in the presence of a critically short telomere

a: Senescence assay on spores having a critically short telomere ( 1 and 2 blocks) and their control (0 block). Each spore-colony from diploids obtained as described in Figure $1 \mathrm{~b}$ (yT136, yT137 and yT138, see Supplementary information) was resuspended to equal concentration and serial 10 -fold dilutions were spotted on rich-media plates. After two days at $30^{\circ} \mathrm{C}$ the process is repeated starting with a mixture of cells from the most concentrated spot. b: Median viability index measured from a taking into account both the capacity to form colonies and the size of the colonies (see Methods). Error bars correspond to standard deviation. Refer to Supplementary Table 1 for further statistical analysis of this data. c: Quantification of the cell cycle distribution of DAPI-stained cells of clones presented in Figure 2a. Exponentially growing cultures of indicated genotype were obtained from the spore colony (sp), $1^{\text {st }}$ or $2^{\text {nd }}$ passage ( $\left.1, \mathrm{p} 2\right)$ and stained with DAPI as described in Methods. Control telomerase positive strains ${ }^{4}$ were included. Data of two independent spores was cumulated. d: spore-colonies were grown overnight in a small volume of YPD, 
diluted to $10^{5}$ cells $/ \mathrm{ml}$ in $100 \mathrm{ml}$ culture volume and grown for 22 hours. Each day, cell culture was diluted back to $10^{5} \mathrm{cells} / \mathrm{ml}$. We report the mean cell concentration counted each day with a hemacytometer of two independent spores of the indicated genotype. Error bars refer to standard deviation. e: data from Figure 1c is reformatted to plot generation time as a function of generations, assuming that by the $3^{\text {rd }}$ day all spores have undergone 35 generations. 

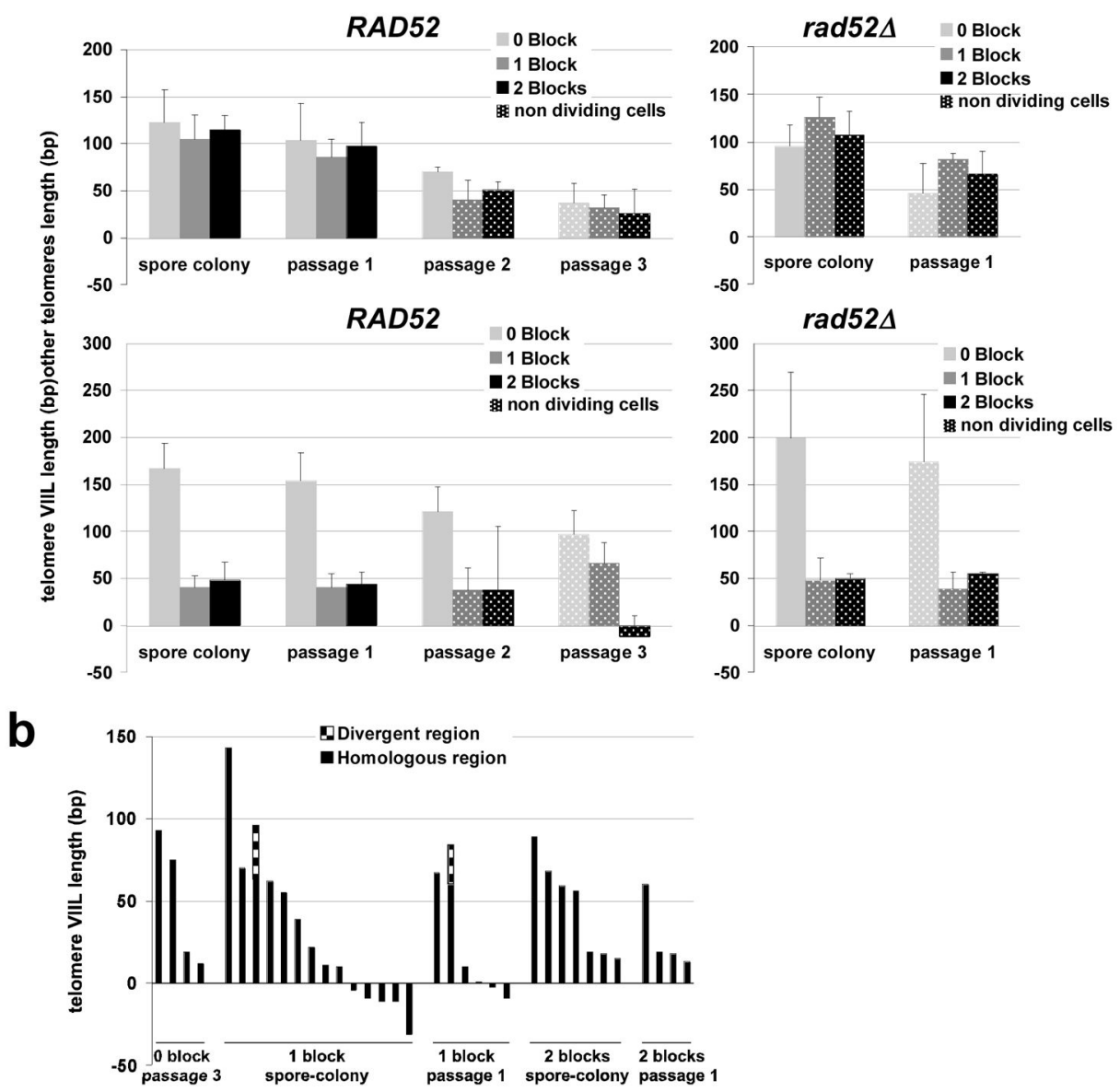

C

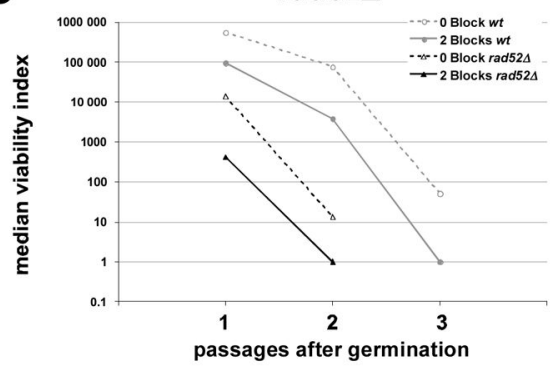

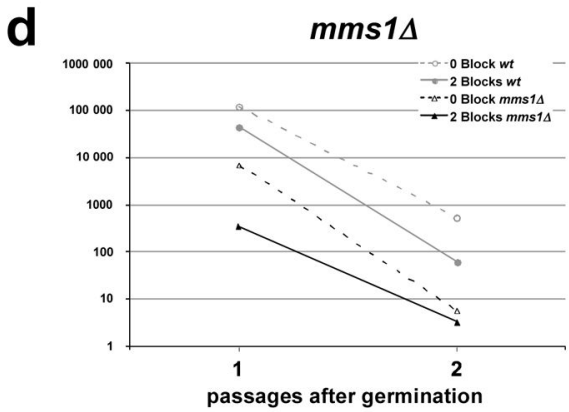

Figure 3. RAD52- and MMS1-dependent growth of pre-senescent cells can be uncoupled from inter-telomeric recombination

a: Genomic DNA was prepared from overnight cultures from successive passages of sister spores $w t$ or rad52 $\Delta$ having excised 1 or 2 blocks of telomeric $\mathrm{TG}_{1-3}$ repeats or no telomeric sequences ( 0 block). Telomere-PCR was performed using oligonucleotide oT157 that targets ADH4 sequences (VII-L telomere) or oT100 that targets $\mathrm{Y}^{\prime}$ telomeres (other telomeres). Estimated mean length of pure $\mathrm{TG}_{1-3}$ repeats measured in at least 5 samples is reported. Error bars refer to standard deviation. b: Telomere PCR of VII-L telomeres from representative indicated samples were cloned and sequenced. We depict length of pure 
$\mathrm{TG}_{1-3}$ repeats. Negative values correspond to telomeres that are truncated in the subtelomeric region. $\mathrm{TG}_{1-3}$ sequences were aligned as described ${ }^{13}$ and divergent repeats were detected. c-d: Diploids described in figure $1 \mathrm{~b}$, containing one allele of the indicated mutations were sporulated and germinated in the presence of galactose to induce the telomere shortening. Median viability was measured as described in Figure 2 using the spots shown in Supplementary Figure 1d and f. Statistical analysis is reported in Supplementary Table 1. 
a

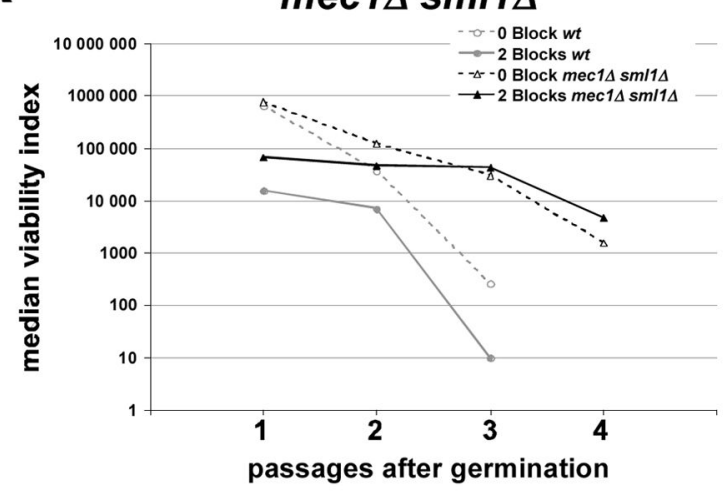

tel1 $\Delta$

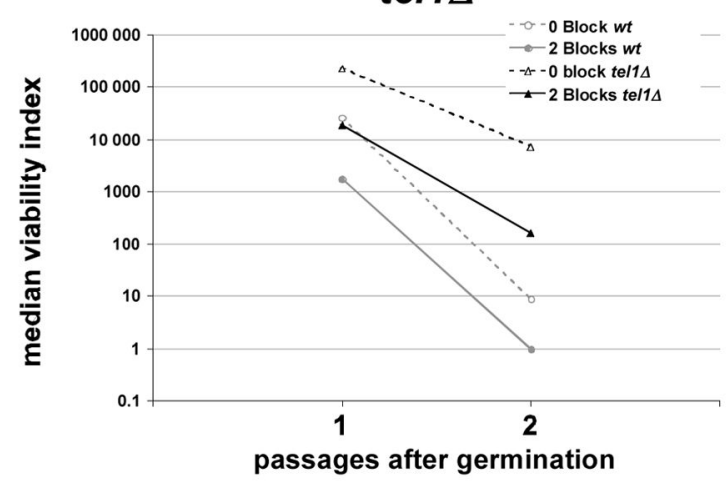

b

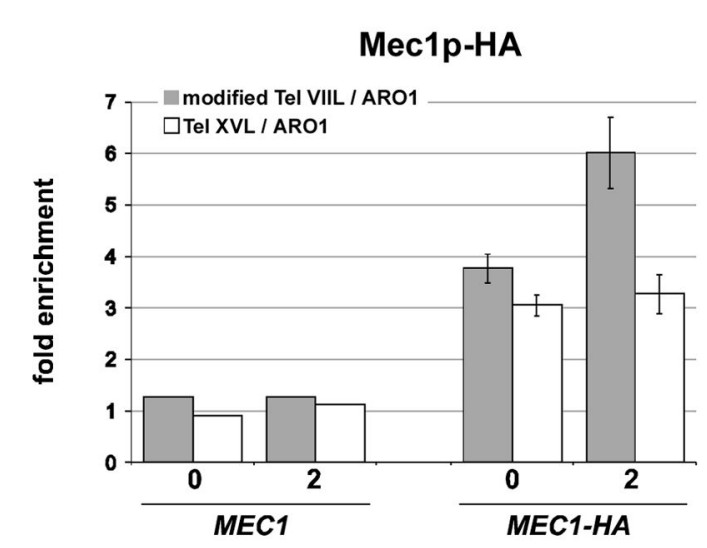

d

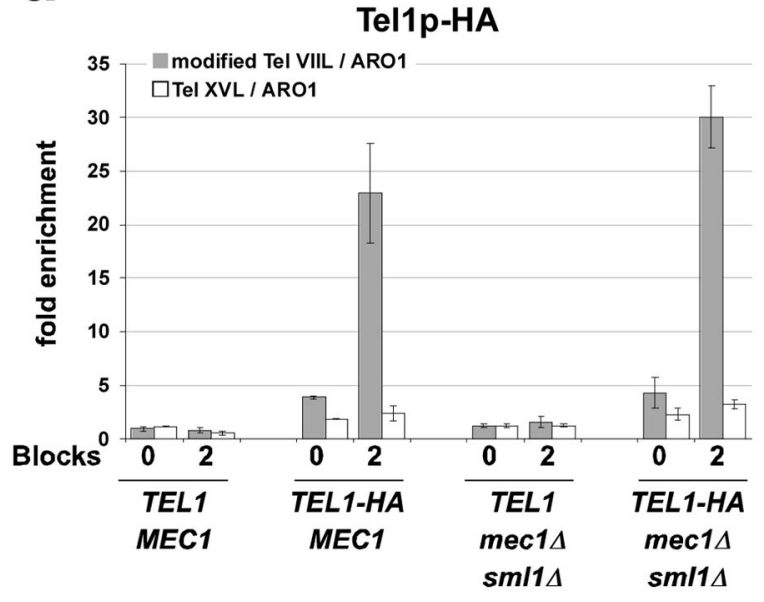

Figure 4. Tel1p promotes senescence and Mec1p signals the very short telomere

a, c: Diploids described in figure $1 \mathrm{~b}$, containing one allele of the indicated mutations were sporulated and germinated in the presence of galactose to induce the telomere shortening. Median viability was measured as described in Figure 2 using spots shown in Supplementary Figure 1g and 1i. Statistical analysis is reported in Supplementary Table 1. b, d: Spore colonies of the indicated genotype, obtained as described in figure $1 \mathrm{~b}$, having (2 blocks) or not ( 0 block) a very short telomere were inoculated in rich medium and grown overnight exponentially. Chromatin was immunoprecipitated with anti-HA antibodies and analysed by quantitative PCR using primers targeting the telomere VII-L, the telomere XV$\mathrm{L}$ and the internal locus $A R O 1$. Enrichment values are calculated as a ratio of the enrichment of a given telomere (Immunoprecipitated / Input) over the ARO1 locus (Immunoprecipitated / Input). 


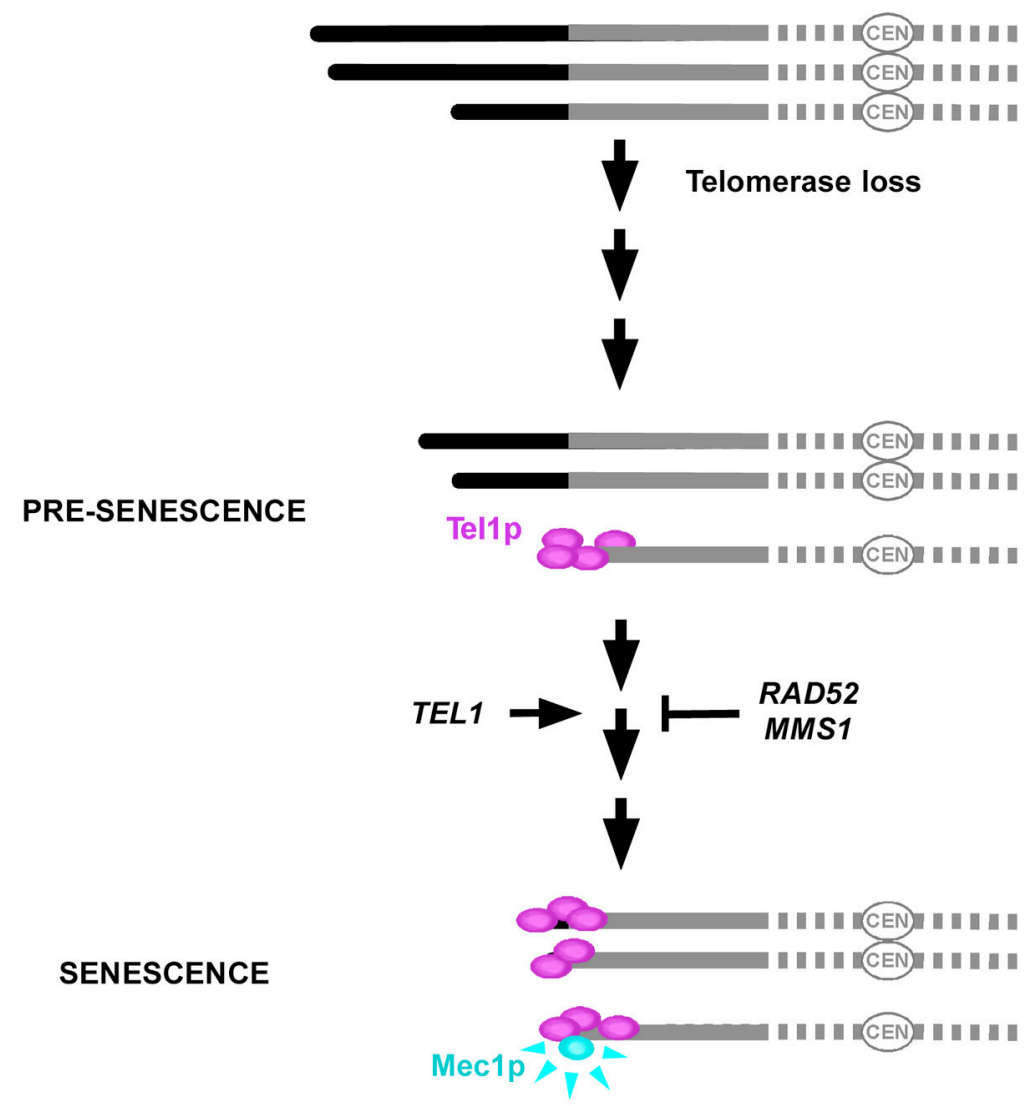

Figure 5. A two-step model for senescence triggered by a single critically short telomere In absence of telomerase, cells containing a critically short telomere are allowed to prolong viability. This allows to define a pre-senescent state that can be modulated by genetic manipulations: RAD52 and MMS1 deletions abrogate this pre-senescent state and TEL1 deletion prolongs it. In the presence of telomerase, this would lead to telomerase recruitment and telomere elongation ${ }^{5,22-24}$. In its absence, telomeres become a substrate for a Rad52p-, Mms1p-dependent processes that protect the telomeres and might abrogate the signaling pathway that activates senescence (e.g. sister chromatid recombination). We demonstrate that Mec1p binds, senses and signals the very short telomere. We speculate that presenescent cells switch to senescent cells when single-stranded subtelomeric sequences are exposed sufficiently to activate Mec1p pathway. This is expected to occur first at the shortest telomere. 